\title{
TOTAL SPONDYLECTOMY IN CHONDROSARCOMA ARISING FROM THE SEVENTH THORACIC VERTEBRA
}

\author{
Bertil Stener, Göteborg, Sweden
}

Total spondylectomy has been performed previously for tumour. In 1966 a complete vertebra (the fourth lumbar) affected by a giant-cell tumour was removed in a two-stage surgical procedure: the body of the vertebra was extirpated two weeks after excision of the posterior parts (Lièvre, Darcy, Pradat, Camus, Bénichou, Attali and Joublin 1968). In 1968, after laminectomy, all remaining parts of three adjacent vertebrae (the eleventh and twelfth thoracic and first lumbar) were removed together with a giant-cell tumour (Stener and Johnsen 1971). Later in the same year the second lumbar vertebra was excised in two patients because of malignant tumour (Hamdi 1969). This paper describes total removal of the seventh and partial removal of the sixth and eighth thoracic vertebrae of a forty-nine-year-old man with a chondrosarcoma arising from the seventh thoracic vertebra.

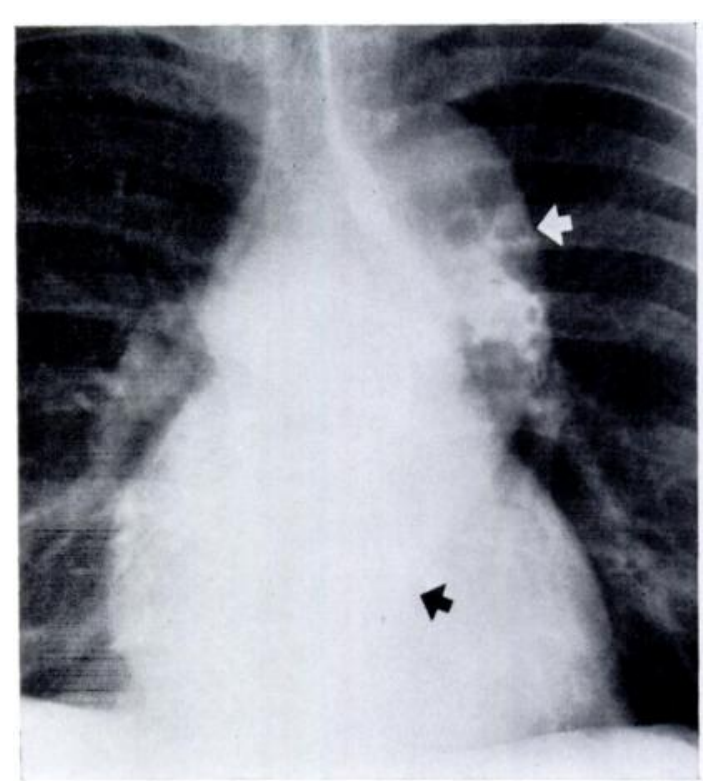

FiG. 1

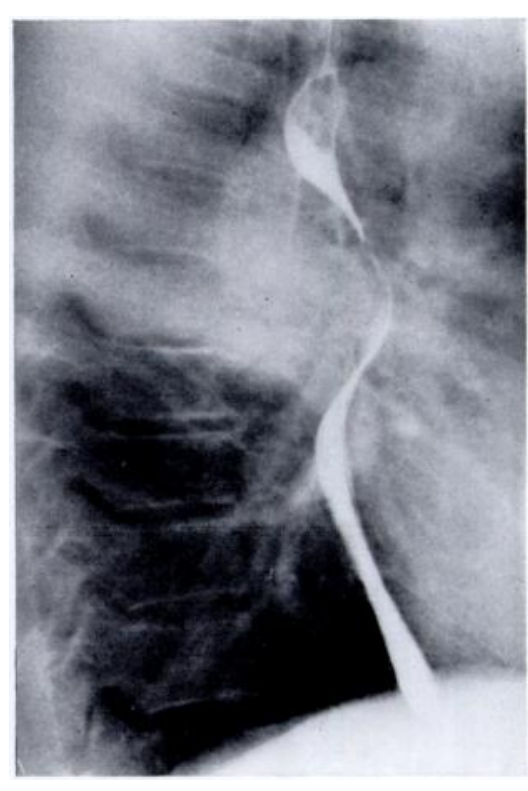

FIG. 2

Figure 1-Antero-posterior radiograph of the chest. The thoracic aorta, marked by arrows, is displaced to the left by the tumour. Figure 2-Lateral radiograph of the chest. The oesophagus, filled with contrast medium, is displaced forward by the tumour.

\section{CASE REPORT}

The patient, a farmer, had for several months suffered pain radiating from the back to the epigastrium, first on the right side then on both sides. Radiographs of the chest showed a tumour of the thoracic spine protruding into the mediastinum. The tumour displaced the aorta to the left (Fig. 1) and the oesophagus forwards (Fig. 2). Tomography suggested that the tumour arose from the seventh thoracic vertebra, the body of which appeared to have been replaced by neoplastic tissue (Figs. 3 and 4). The tumour seemed to have spared the 


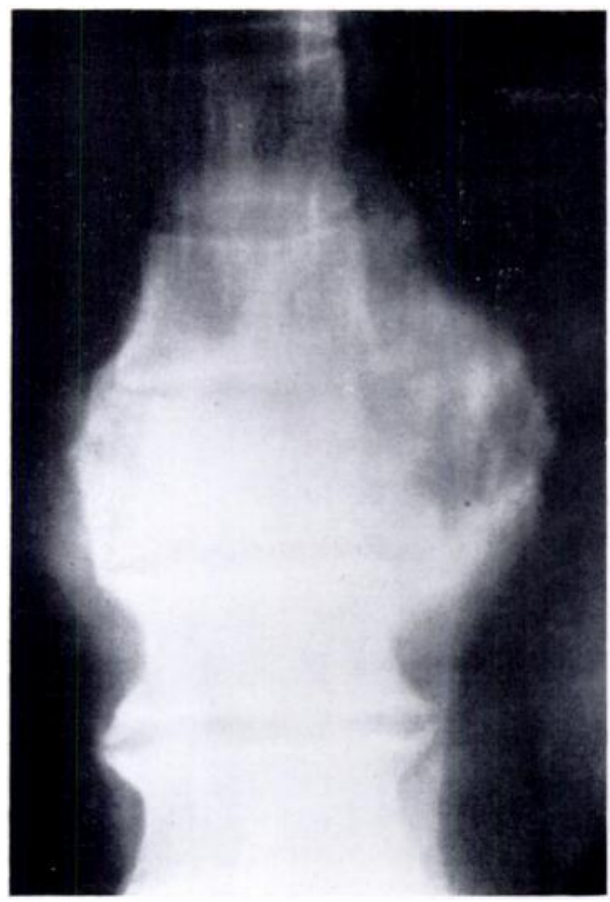

FIG. 3

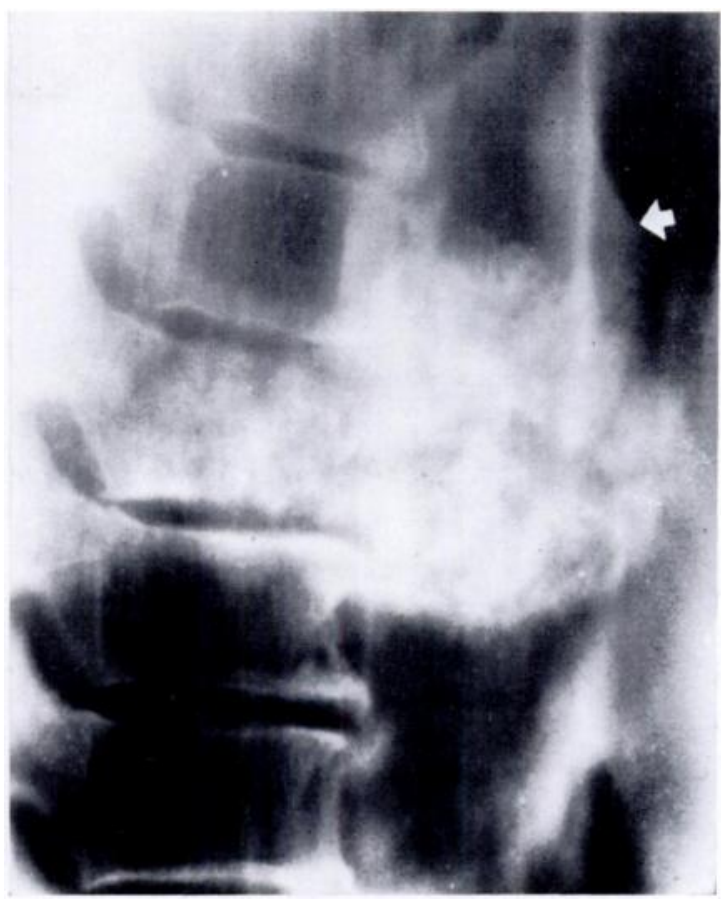

Fig. 4

Figure 3-Frontal tomograph of the thoracic spine. Tumour expanding from the body of the seventh vertebra. Figure 4-Sagittal tomograph of the thoracic spine. The body of the seventh vertebra appears to have been replaced by neoplastic tissue. The tumour protrudes into the mediastinum. Arrow marks the bifurcation of the trachea.

adjacent intervertebral discs, having instead expanded outside the vertebra, chiefly in an anterior direction. The bulk of the tumour, rounded in shape, lay in the mediastinum, just below the bifurcation of the trachea (arrow in Fig. 4). Aortography showed that several of the right posterior intercostal arteries were displaced anteriorly by the tumour. The tumour itself appeared sparsely vascularised. The patient showed no sign of compression of the spinal cord, but gas myelography revealed a complete block at the level of the seventh thoracic vertebra, suggesting that the tumour bulged posteriorly from the body of this vertebra (Fig. 5). Radiographic appearances suggested that the tumour was a well differentiated chondrosarcoma.

Pre-operative considerations-The patient suffered from constant pain, and the location of the tumour meant that paraplegia and eventual death could be expected. Because of this, and of the good physical condition of the patient, it was decided to attempt extirpation of the tumour. The main concern in the planning of the operation was the

FIG. 5

Gas myelograph. Displacement of the spinal cord (white arrow') and complete block at the level of the seventh vertebra (black arrow) suggesting that the tumour protrudes posteriorly.

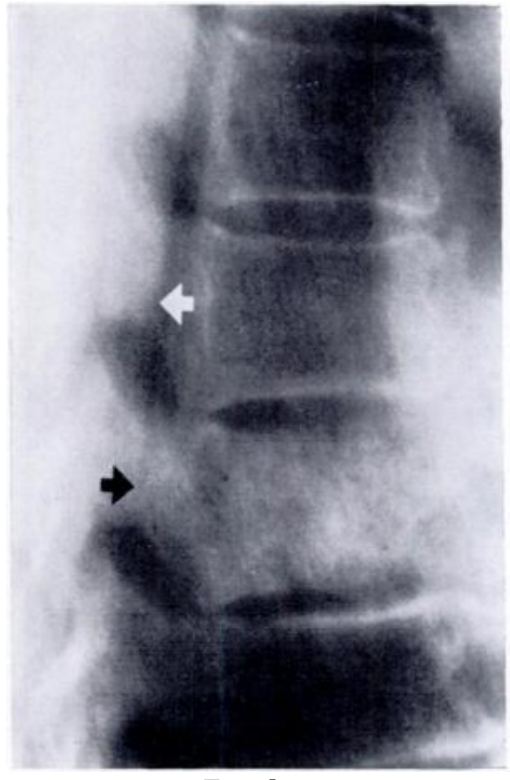

FIG. 5 
risk of impairing the blood supply to the spinal cord by the necessary sacrifice of the seventh thoracic nerve roots on either side. According to Lazorthes, Poulhes, Bastide, Roulleau and Chancholle (1958) the thoracic part of the spinal cord depends for its adequate nutrition on one or several "radicular" arteries, branches of the posterior intercostal arteries accompanying the nerves to the cord. The level of the most important of these arteries is subject to much individual variation. Thus one had to reckon with the possibility that in this patient the thoracic portion of the spinal cord depended mainly on an artery accompanying one of the seventh nerve roots. However, it was felt that this calculated risk had to be taken in view of the certainty of paraplegia if operation was not performed. (The radicular arteries of the spinal cord were too small to be recognised in the aortographs.)

Operation-The patient was placed prone on a Wilson frame in order to avoid compression of the inferior vena cava. A midline incision over the thoracic spine was prolonged at the thoraco-lumbar junction to both sides in an oblique latero-inferior direction (Fig. 6). The trapezius, the rhomboids and the superior part of the latissimus dorsi were severed bilaterally from the thoracic spinous processes. The erectores spinae muscles were detached from the spinous processes, the laminae and the medial part of the transverse processes of the third to the tenth thoracic vertebrae on each side. These muscles were then severed transversely on a level with the seventh thoracic vertebra. After subperiosteal resection of both seventh

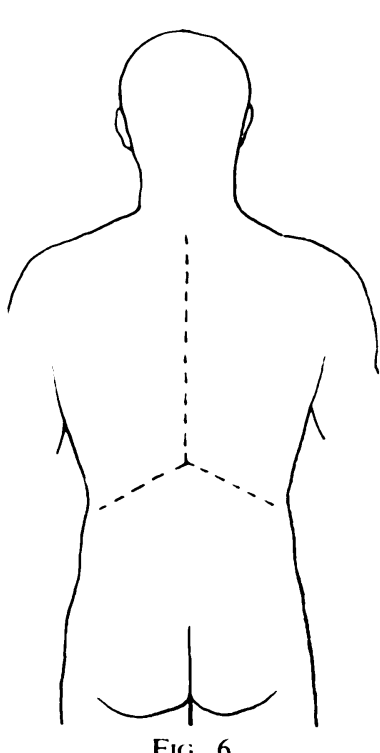

FICi. 6

Incision of the skin. ribs, from the costo-transverse joint to the mid-axillary line, both the pleural cavities were opened. In order to gain exposure within the thorax, the fifth, sixth and eighth ribs were transected on each side just lateral to the costo-transverse joint; care was taken not to damage unnecessarily intercostal nerves and vessels. Then the spinal cord enclosed in the theca was exposed from behind by removal of the spinous process and the inferior articular processes of the sixth thoracic vertebra, of the spinous process, the laminae, the superior and inferior articular processes, and the transverse processes of the seventh thoracic vertebra, and of the superior articular processes of the eighth thoracic vertebra (Fig. 7, middle). No tumour tissue was seen in the cut posterior surface of the pedicles of the seventh thoracic vertebra. Instead, the tumour bulged in a posterior direction from the body of this vertebra, particularly on the right side so that the spinal cord was displaced backwards and to the left.

With the posterior vertebral resection completed, the operation was continued within the thorax. As had been shown radiographically, the major part of the tumour lay in the posterior mediastinum, anterior to the bodies of the sixth, seventh and eighth

vertebrae (Fig. 4). The tumour appeared well delimited with no apparent infiltration of important mediastinal organs. The intervertebral discs between the fifth and sixth and between the eighth and ninth vertebrae were identified and exposed. Then, starting at the anterior edge of these discs, oblique osteotomies were done through the bodies of the sixth and eighth vertebrae (Fig. 7, middle). Using a Gigli saw, the osteotomy was directed postero-inferiorly through the body of the sixth and postero-superiorly through the body of the eighth vertebra. The last part of the osteotomy was done with a chisel directed transversely from one side to the other. Because all posterior parts of the seventh vertebra except the pedicles had been removed earlier during the operation, the completion of the osteotomies meant that the tumour specimen became loose so that it could be moved forward enough to release the pressure against the spinal cord. The sheath of the seventh nerve root was ligated and severed on either side, and the thecal sac was freed by blunt dissection from a layer of fibrous tissue covering the 
posteriorly protruding part of the tumour. The specimen was then pushed forward enough for the left pedicle of the seventh thoracic vertebra to pass anterior to the spinal cord while the specimen was rotated around the axis of the body with the posterior part being turned to the right and the anterior to the left. The specimen was then moved backwards out through the right thoracotomy opening.

The only structure thereafter bridging the gap in the thoracic spine was the spinal cord enclosed in the thecal sac (Fig. 7, middle). An assistant held the superior and inferior portions of the thoracic spine fixed with instruments gripping spinous processes.

For reconstruction of the spine two "A.O."* plates of stainless steel were used, measuring $20 \times 1.6 \times 0.6$ centimetres and provided with twelve holes (Fig. 7, left). Having been bent to fit the thoracic curve the plates were fastened, one on each side of the spinous processes, with double, 1 millimetre thick, stainless steel wires to the transverse processes of the third to the sixth and the eighth to the tenth vertebrae (Fig. 7, middle and top right). At the upper and lower ends the plates were secured to each other by wires. Thereafter, one block of bone was taken from the posterior part of each ilium, using separate incisions. The two blocks of bone were given a suitable form and were then put together (Fig. 7, bottom right) and inserted between the bodies of the sixth and eighth vertebrae (Fig. 7, middle). By pressing the boneblocks backwards a good contact was obtained between the obliquely cut ends of the blocks and the obliquely cut vertebral bodies. A transverse bore-hole was made through the superior and inferior ends of the blocks and through the bodies of the fifth and ninth vertebrae. Via these boreholes the blocks were fastened to the spine with silk threads (Fig. 7, middle). Further

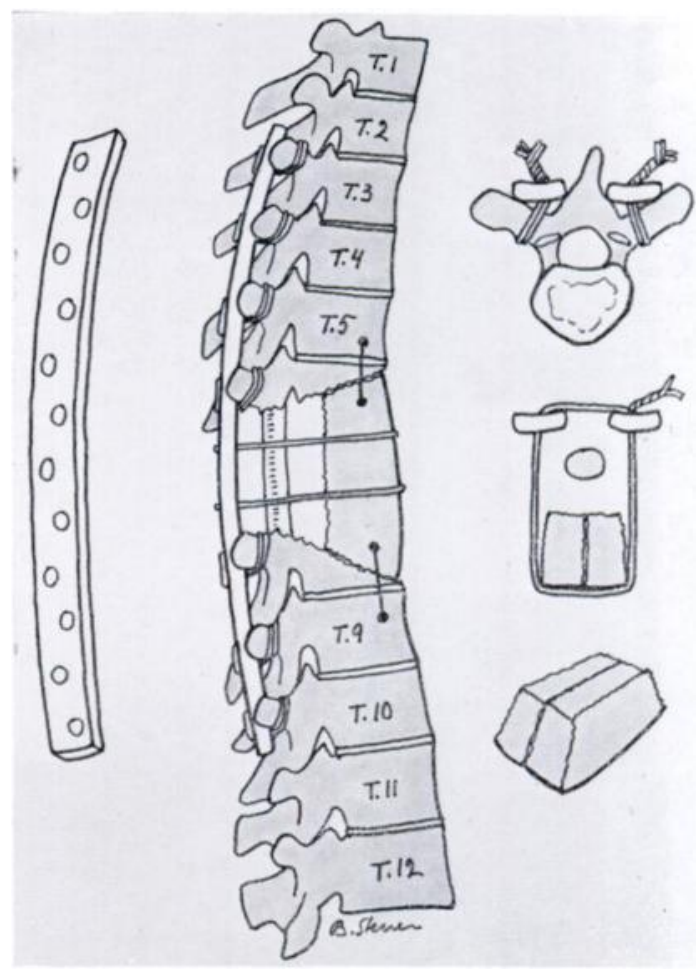

FIG. 7

Reconstruction of the spine. The antero-inferior half of the body, the inferior articular processes, and the spinous process of the sixth vertebra, all of the seventh. and the antero-superior half of the body and the superior articular processes of the eighth have been removed along with the tumour. Two plates have been fastened with double steel wires to the transverse processes of the third to the sixth and the eighth to the tenth vertebrae (middle and top right). Two iliac boneblocks with obliquely cut ends have been put together (bottom right) and inserted between the obliquely cut bodies of the sixth and eighth vertebrae (middle). The blocks have been fastened to the spine with silk threads passed through holes in the blocks and the vertebrae. Further fixation has been provided by two steel wires fastened to the plates and gripping the bone-blocks (middle and middle right).

fixation was provided by two steel wires

which, gripping around the anterior, cortical surface of the blocks, were fastened to the "A.O." plates (Fig. 7, middle and middle right). A piece of cancellous bone from the ilium laid in contact with the posterior surface of the blocks was fastened by a thread of chromic catgut passed around the blocks. After reconstruction of the spine two suction tubes were inserted into each pleural cavity, one anteriorly and one posteriorly. The thoracotomy wounds were closed and the trapezius, the rhomboids and the latissimus dorsi were reattached to the spine. Finally the skin was closed with steel sutures.

\section{* The Swiss Association for Osteosynthesis.}




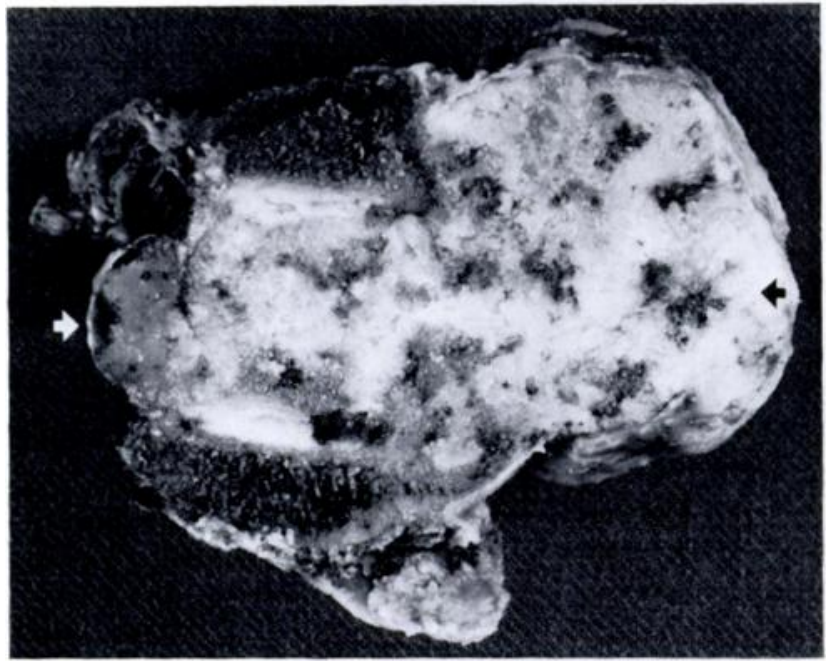

FIG. 8

The specimen transected, almost sagitally. The tumour has replaced the body of the seventh vertebra, whereas the adjacent intervertebral discs have been largely spared. The black arrow indicates the anterior, mediastinal part of the tumour; the white arrow points to the posteriorly protruding part which caused displacement of the spinal cord (Fig. 5).

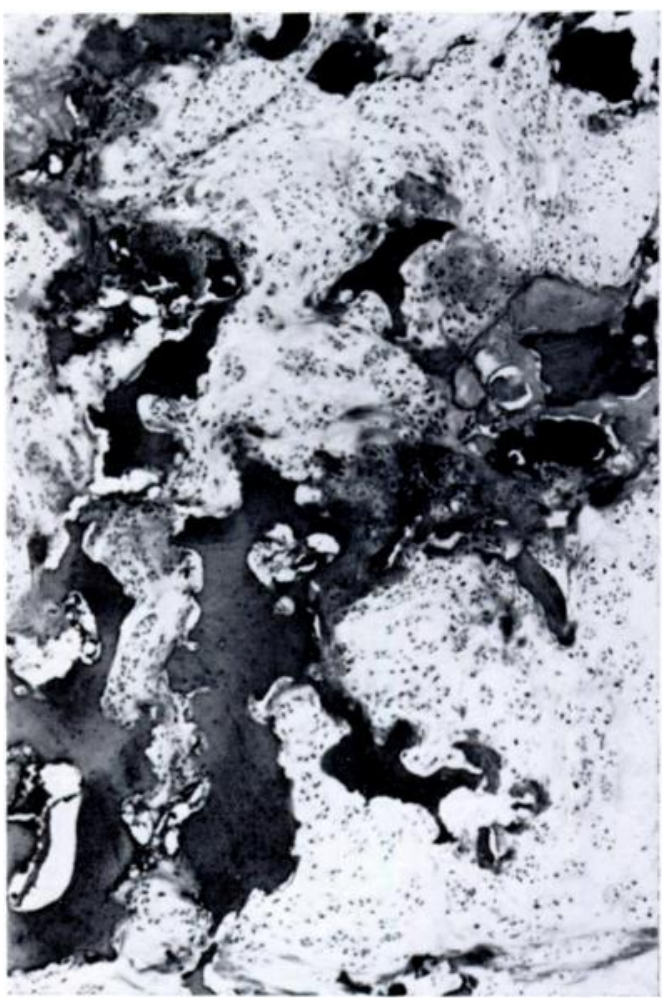

FIG. 9

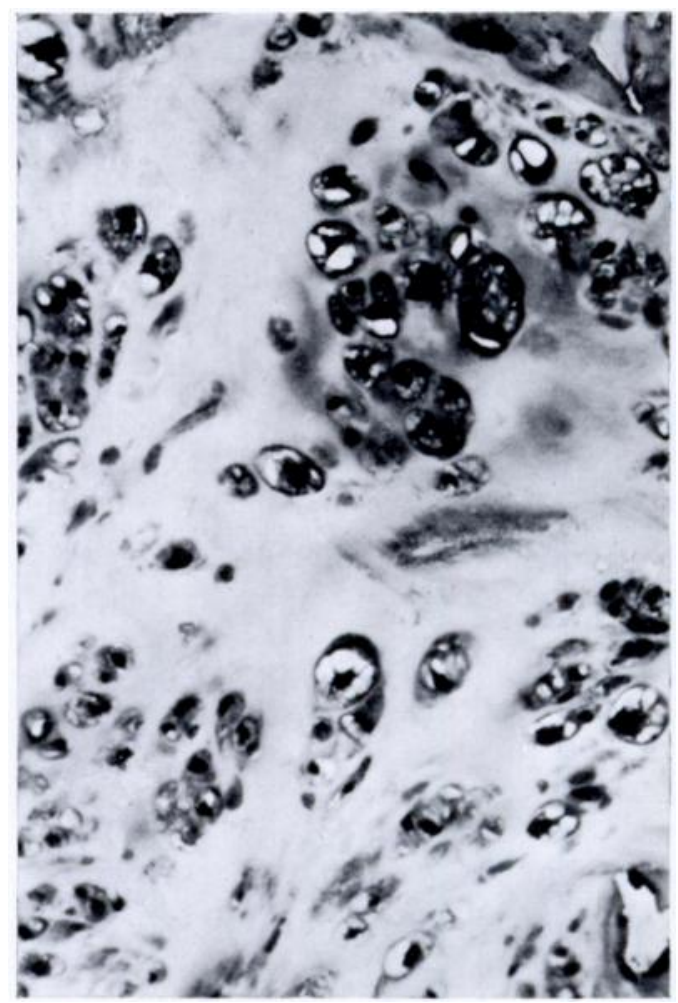

Fig. 10

Histological appearance of tumour. Figure 9-Cartilaginous tissue surrounds an archipelago of partly necrotic bone (dark spots within the tumour in Fig. 8). (Van Gieson, $\times 30$.) Figure 10-Cartilaginous tissue showing cellular and nuclear pleomorphism. (Haematoxylin and eosin, $\times 190$.) 
Operative specimen-The specimen is shown transected in a nearly sagittal plane in Figure 8. The cut surface of the specimen corresponds fairly well with the tomographic picture in Figure 4. The tumour has replaced the body of the seventh vertebra, but the adjacent intervertebral discs have largely been spared. The part of the tumour that bulged backwards (arrow in Figure 8) was obviously the reason for the displacement of the spinal cord shown by gas myelography (Fig. 5). The consistency of the tumour was hard. Its colour in the cut surface was bluish-grey with irregular dark red spots apparently corresponding to islands of cancellous bone.

Histological examination (Dr L. Angervall) showed a fairly well differentiated, moderately cellular chondrosarcoma with rather marked cellular and nuclear pleomorphism (Figs. 9 and 10). Some cells were plump and had an irregular hyperchromatic nucleus. Several cells contained two nuclei. A few mitotic figures were seen.

Progress after operation-After operation the patient was put in a plaster bed. A wedge of wood ( 25 degrees) was used to facilitate tilting of the bed to the left and the right sides at regular intervals. Because of the extensive bilateral thoracotomy it was considered advisable to do a tracheostomy and connect this to a respirator. Five days after operation there was bleeding from the gastro-intestinal tract. A diagnosis of a stress ulcer was made, and treatment with blood transfusions and atropine was instituted. At first this treatment seemed to be successful. However, twelve days after the operation, profuse bleeding started again, and after three more days of conservative treatment operation was decided on. A prepyloric ulcer with a bleeding artery was found. The bleeding was stopped by a few suture ligatures. Then pyloroplasty, vagotomy and gastrostomy were performed.

After this operation the condition of the patient gradually improved. Oral feeding could be started after about one week. Then the patient was trained to be independent of the respirator, which was discontinued permanently five weeks after the initial operation. Four weeks later the patient was turned to a prone position for inspection of the back and removal of the stitches. All wounds had healed primarily

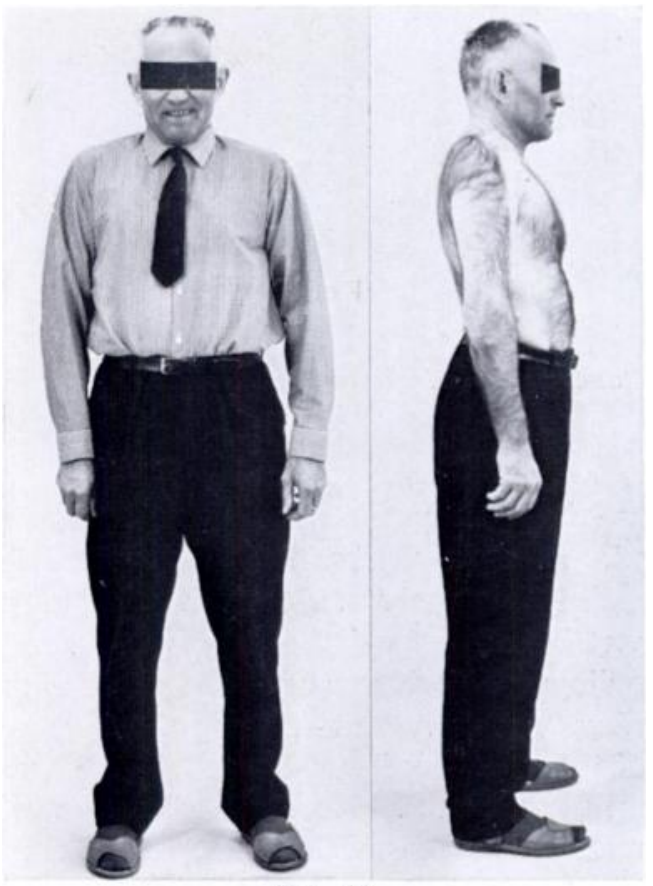

FIG. 11

Patient fifteen months after operation able to stand and walk without external support.

except for a small area of skin necrosis at the lower end of the midline incision where this had been prolonged to either side in a latero- inferior direction (Fig. 6).

Three and a half months after operation a brace controlling forward bending was substituted for the plaster bed. About a month later gradual training to an upright position by means of a tilt table was started. Eleven months after operation the patient was allowed to start walking in a walking chair. At present, four months later, he is able to stand (Fig. 11) and walk without external support but still prefers to use crutches mainly because he is afraid of falling.

Radiographic progress - Six months after operation tomography (Fig. 12) suggested that fusion had taken place between the iliac grafts and the bodies of the sixth and eighth vertebrae. Radiographic examinations repeated thereafter at monthly intervals have consistently shown unchanged position of the grafts, the "A.O." plates and the wires, despite increasing physical 

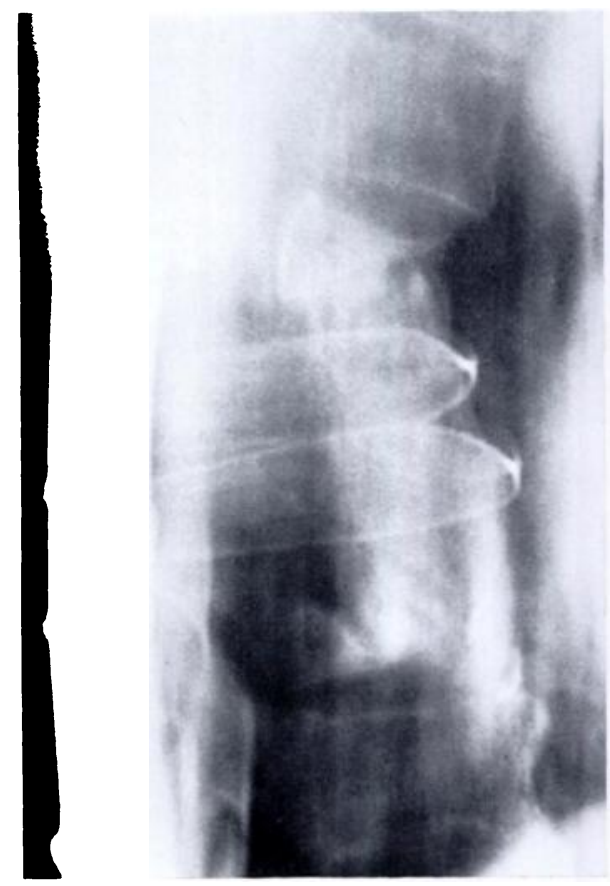

Fig. 12

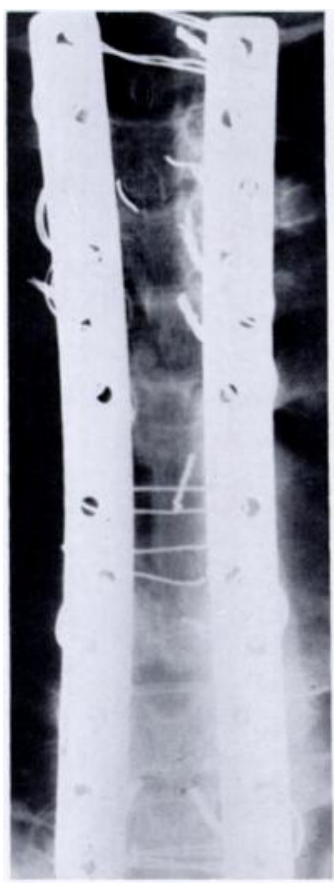

Fig. 13

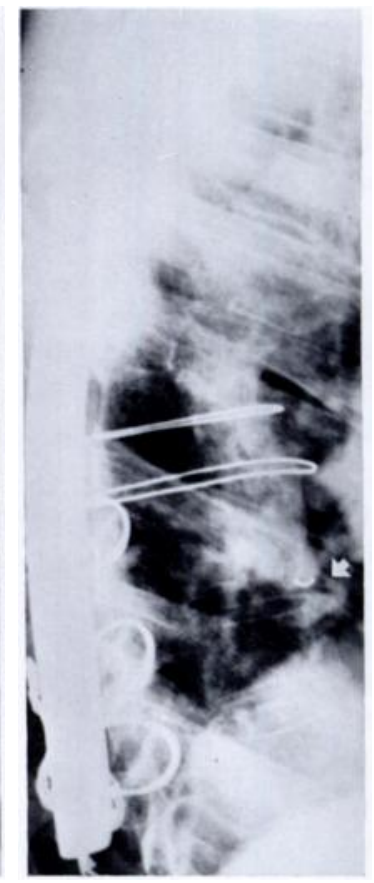

Figure 12-Sagittal tomograph six months after operation suggesting fusion between the iliac grafts and the bodies of the sixth and eighth vertebrae. Figure 13-Antero-posterior and lateral radiographs fifteen months after operation. Block-vertebra created from iliac grafts and remaining parts of sixth and eighth vertebrae. Arrow indicates osteophytes connecting grafts to the ninth vertebra.

activity of the patient. Radiographs taken fifteen months after operation are shown in Figure 13. A block-vertebra has been created from the iliac grafts and the bodies of the sixth and eighth vertebrae. At the lower end the grafts have been connected to the body of the ninth vertebra by osteophytes (arrow in Figure 13) bridging the intervening disc. There is no radiographic evidence of metastasis or of recurrence of the tumour.

Neurological progress-During the early period after operation, assessment of the function of the spinal cord was hampered by the necessity of administering analgesics, sedatives and muscle-relaxants. No signs of any grave ischaemic lesion were observed, however: tone remained in the legs, the reflexes were normal, weak active movements were observed, and the patient reacted to pain stimuli. During the episodes of gastric bleeding when blood pressure and haemoglobin sometimes fell to very low values, no voluntary motor activity could be demonstrated in the lower limbs. Since then, the motor and sensory function of the lower limbs has gradually improved, so that now, fifteen months after the operation, they are largely restored. He moves the hips, knees and ankles with good strength and perceives touch in all parts of the legs; he feels vibration in the tibiae and can distinguish between dorsal and plantar flexion of the great toes. The limbs are rather spastic: the Babinski sign is present and the knee and ankle jerks are exaggerated. The spasticity is, however, much less pronounced now than during early rehabilitation; ankle clonus which could then be elicited is now absent. After operation the patient had an indwelling catheter for five weeks. Since that time he has had full control of the bladder.

\section{COMMENT}

This operation obviously carried a great risk of failure. It seemed justified, however, because of the risk to life. Chondrosarcoma is radio-resistant and cannot be eradicated except 
by surgical excision (Dahlin 1967). Of course it is too early to know whether the patient has indeed been cured, but considering the low tendency of chondrosarcoma to metastasise (Dahlin 1967) the patient should have a reasonably good chance of long survival. A definite cure cannot be claimed until at least ten years have passed without the appearance of recurrence or metastasis (Dahlin 1967).

The method used for reconstruction of the spine after extirpation of the tumour needs a few comments. The two "A.O." plates which were wired to the transverse processes of the third to the sixth and the eighth to the tenth vertebrae provided good stability. Being relatively strong, the transverse processes of the thoracic vertebrae are well suited for this type of fixation. The wires were passed between the transverse process and the neck of the corresponding rib. Inserted in this way the wires are prevented by costo-transverse ligaments from slipping laterally over the tip of the transverse process. Such lateral displacement was also guarded against by wiring the two plates to each other at the upper and lower ends (Fig. 13).

The principle behind the bony rebuilding of the spine was to insert iliac grafts between the cut bodies of the sixth and eighth vertebrae in such a way that an intimate contact, under some pressure, was obtained between surfaces of cancellous bone, those of the two vertebrae involved and those of the grafts (Fig. 7). The posterior part of each ilium served as an excellent source of large cancellous bone-blocks partly reinforced by cortical laminae. The latest radiographs (Fig. 13), taken fifteen months after the operation, when the patient had been very active for a long time, demonstrate that the grafts have become incorporated in the spine.

\section{SUMMARY}

1. A forty-nine-year-old man had a chondrosarcoma arising from the body of the seventh thoracic vertebra. The tumour protruded into the mediastinum and also into the spinal canal where it displaced the spinal cord.

2. At operation all the seventh thoracic vertebra and parts of the sixth and eighth were removed together with the tumour. The thoracic spine was reconstructed by inserting two iliac bone-blocks between the cut bodies of the sixth and eighth vertebrae and by wiring two strong "A.O." plates to the transverse processes of the third to the sixth and the eighth to the tenth vertebrae.

3. The patient was nursed in a plaster-of-Paris bed for three and a half months.

4. One year and three months after operation, the patient was walking and well, with no signs of recurrence or metastasis. Radiographs showed that a block-vertebra had been created from the iliac grafts and the two cut vertebrae.

\section{REFERENCES}

Dahlin, D. C. (1967): Bone Tumors. Second edition. Springfield, Illinois: Charles C. Thomas, Publisher.

Hamdi, F. A. (1969): Prosthesis for an Excised Lumbar Vertebra: A Preliminary Report. The Canadian Medical Association Journal, 100, 576.

Lazorthes, G., Poulhes, J., Bastide, G., Roulleau, J., and Chancholle, A.-R. (1958): La vascularisation artérielle de la moelle. Neuro-Chirurgie, 4, 3.

lièvre, J.-A., Darcy, M., Pradat, P., Camus, J.-P., Bénichou, C., Attali, P., and Joublin, M. (1968): Tumeur a cellules géantes du rachis lombaire, spondylectomie totale en deux temps. Revue du rhumatisme et des maladies ostéo-articulaires, 35, 125.

Stener, B., and Johnsen, O. E. (1971): Complete Removal of Three Vertebrae for Giant-cell Tumour. Journal of Bone and Joint Surgery, 53-B, 278. 